\title{
Properties of the Gold Sequence P.R.K. Applied to Acoustic CT
}

\author{
Yukio Fukayama, Takuya Saiki, Akitoshi Ozawa and Yuki Hotani \\ Dept. of Electronic Control, Niihama National College of Technology \\ 7-1 Yakumo-cho, Niihama, 792-8580 Japan
}

E-mail: fukayama@ect.niihama-nct.ac.jp

\begin{abstract}
In this report, at first, an algorithm for CT based on the MAP (maximum a posteriori) estimation is derived for the systems, and a matched filter with complex absolute detection is introduced for the optimal signal processor to be free from non-linear phase shift. Then, to reduce the measuring time, the Gold sequence P.R.K. (Phase Reversal Keying) is proposed to the signal, since it provide sharp autocorrelations and low cross-correlations, being useful for simultaneous time-of-flight identifications on many acoustic paths. Finally, the total performance of the proposed methods is illustrated.
\end{abstract}

\section{Introduction}

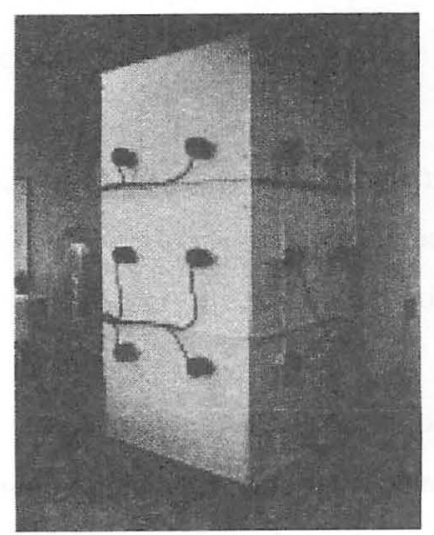

Fig.1 An Acoustic CT system for a duct
A typical acoustic CT (Computed Tomography) system for 3-D distribution of gas temperature[2], illustrated in Fig.1, is equipped with 24 transducers, which send and receive acoustic signals. Thus, 216 of paths on which acoustic time-of-flight are observed are formed up, combining with these transducers. In ordinary cases, the CT systems are assumed to consist of 10 to 30 transducers and 200 to 2000 of acoustic paths.

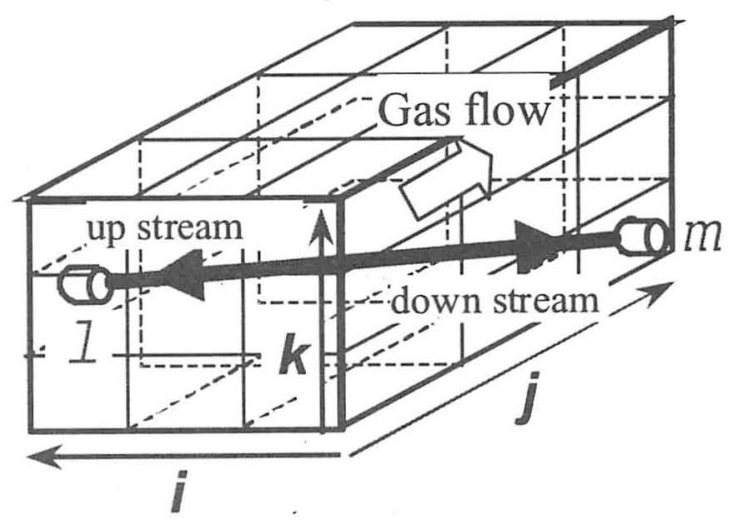

Fig.2 A gas duct, fallen sideways

As shown in a view of fallen sideways, Fig.2, the CT system include, in spatially, $n$-divided cells of the duct inside, with referring the observed time-of-flights of both up and down stream on the totally $p$ acoustic paths among the transducers. Gas flow velocity to the $j$-direction, from bottom to top of the duct, is also assumed in the system. The seeking distributions of gas temperature and flow are immediately calculated from the 
estimated states, sound speed and gas flow in each cells, knowing the physical properties of the gas.

The purposes of this research are to reduce the measuring time over many acoustic paths, as well as to improve accuracy of the estimated distributions. Thus the Gold sequence, which has sharp autocorrelations and low cross-correlations, shall be discussed in this report.

\section{Estimation of Distributions}

Seeking state $x(k) \in \mathbb{R}^{2 n}$ at $k$, which means the discretized time at $\tau=k \Delta \tau$ with sampling interval $\Delta \tau$, is defined as follows:

$$
\begin{aligned}
\boldsymbol{x}(k)= & \left(\begin{array}{llllll}
x_{1} & \cdots & x_{n} & x_{n+1} & \cdots & x_{2 n}
\end{array}\right)^{\mathrm{T}} \\
& \text { sound speed }
\end{aligned}
$$

where $x_{q}$ and $x_{n+q}$ respectively denote the sound speed and gas velocity to the $j$-direction at $q$-th $(q=1, \cdots, n)$ cell.

Similarly observation $y(k) \in \mathbf{R}^{2 p}$ is defined as follows:

$$
\begin{aligned}
y(k)= & \left(\begin{array}{llllll}
y_{1} & \cdots & y_{p} & y_{p+1} & \cdots & y_{2 p}
\end{array}\right)^{\mathrm{T}} \\
& \text { down stream up stream }
\end{aligned}
$$

where $y_{r}$ and $y_{p+r}$ respectively denote the acoustic time-of-flights in down and up stream on the $r$-th $(r=1$, $\cdots, p)$ path.

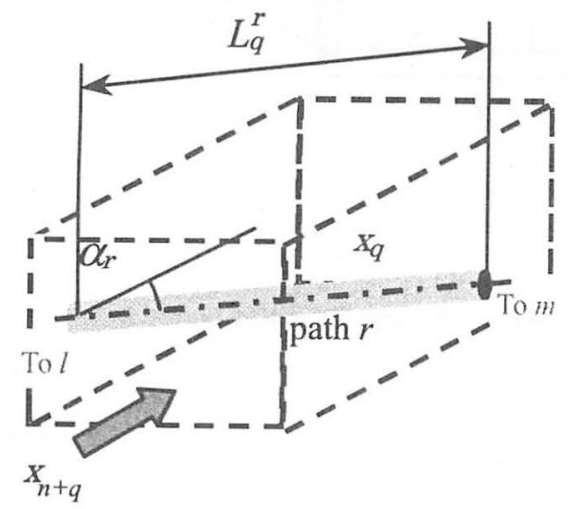

Fig.3 Contribution to a path from a cell

Then the observation process of the system is modeled with non-linear observation equation $h(x) \in \mathbf{R}^{2 p}$ and random noise $w(k) \in \mathbf{R}^{2 p}$ as follows:

$$
y(k)=\boldsymbol{h}(x(k))+\boldsymbol{w}(k)
$$

where $h(x)$ consists of total contributions to the acoustic time-of-flights in down and up stream on the $r$-th path from the each cell. These contributions are described, referring Fig.3, with the sound speed $x_{q}$, gas velocity $x_{n+q}$, angle made by the flow and the acoustic path and length $L_{q}^{r}$ of the path in the $q$-th cell as follows:

$h(x)=\left(\begin{array}{lllll}\cdots & h_{r} & \cdots & h_{p+r} & \cdots\end{array}\right)^{\mathrm{T}}$

$\left\{\begin{array}{l}h_{r}=\sum_{q=1}^{n} \frac{L_{q}^{r}}{x_{q}+x_{n+q} \cos \alpha_{r}} \text { (down stream), } \\ h_{p+r}=\sum_{q=1}^{n} \frac{L_{q}^{r}}{x_{q}-x_{n+q} \cos \alpha_{r}} \quad \text { (up stream) }\end{array}\right.$

Then the following system equation at $k$ is assumed.

$$
x(k+1)=\mathrm{F}(k) x(k)+u(k)
$$

where $\mathrm{F}(k) \in \mathbf{R}^{2 n \times 2 n}$ and $\boldsymbol{u}(k) \in \mathbf{R}^{2 n}$ denote the transition matrix, which should be regular, and the disturbance to the system respectively. Matrix, $\mathrm{F}(k)$ should be given considering the system dynamics, however, it can be assumed to be the identity matrix for smoothing.

In addition, $\boldsymbol{w}(k), \boldsymbol{u}(k)$ and $\boldsymbol{x}(0)$ are practically regarded as Gaussian with the following properties:

$\mathbf{E}\{\boldsymbol{u}(k)\}=\overline{\boldsymbol{u}}(k), \mathbf{E}\{\boldsymbol{w}(k)\}=\overline{\boldsymbol{w}}(k)$,

$\mathbf{E}\{x(0)\}=\bar{x}_{0}$

$\mathbb{E}\left\{\left(\begin{array}{c}w(k)-\bar{w}(k) \\ u(k)-\bar{u}(k)\end{array}\right)\left(\begin{array}{c}\boldsymbol{w}(l)-\bar{w}(l) \\ \boldsymbol{u}(l)-\bar{u}(l)\end{array}\right)^{\mathrm{T}}\right\}=\left(\begin{array}{cc}\mathrm{W}(k) & \mathrm{O} \\ \mathrm{O} & \mathrm{U}(k)\end{array}\right) \delta_{k, l}$

$\mathbf{E}\left\{\left(\begin{array}{c}w(k)-\bar{w}(k) \\ x(0)-\bar{x}_{0}\end{array}\right)\left(x(0)-\bar{x}_{0}\right)^{\mathrm{T}}\right\}=\left(\begin{array}{c}\mathrm{O} \\ \mathrm{P}_{0}\end{array}\right)$

where $\bar{u}(k), \mathrm{U}(k), \bar{w}(k), \mathrm{W}(k), \bar{x}_{0}$ and $\mathrm{P}_{0}$, in order, denote mean vectors and co-variance matrices of $w(k)$, $u(k), x(0)$.

These six parameters for (6) to (8) usually assumed to be zero means and diagonal co-variances which can be tuning parameters for response of measurement in actual situations.

As introduced in the previous report[2], the seeking state can be estimated with the following algorithm that is applied an iterated method of the MAP (maximum a posteriori) criterion. Estimation of $\hat{x}(k \mid k)$ is performed with a measurement updating procedure that is applied an 
initial condition and the convergence decision with allowance $\varepsilon$ as follows:

$$
\begin{aligned}
& \hat{x}^{(0)}=\hat{x}(k \mid k-1) \\
& \hat{x}(k \mid k)=\hat{x}^{(j)} \quad\left(\text { under }\left\|\hat{x}^{(j)}-\hat{x}^{(j-1)}\right\|<\varepsilon\right)
\end{aligned}
$$

where $\hat{\boldsymbol{\theta}}_{k / n}$ and super script ${ }^{(j)}$ denote the estimated values of - at $k$ given $\sigma$-field associated with observations up to $n$ and values at the $j$-th iteration respectively.

The procedure with iterations is as follows:

$$
\hat{x}^{(j+1)}=\hat{x}^{(j)}-\Pi_{k}^{-1}\left(\hat{x}^{(j)}\right) \gamma_{k \mid k}\left(\hat{x}^{(j)}\right)
$$

where the following quantities are defined:

$$
\begin{aligned}
& \Pi_{k}(x) \equiv \mathrm{P}^{-1}(k \mid k-1)+\mathrm{H}^{\mathrm{T}}(x) \mathrm{W}^{-1}(k) \mathrm{H}(x) \\
& \gamma_{k \mid k}(x)=-\mathrm{P}^{-1}(k \mid k-1)(x-\hat{x}(k \mid k-1)) \\
& +\mathrm{H}^{\mathrm{T}}(x) \mathrm{W}^{-1}(k)(y(k)-h(x)-\bar{w}(k)) \\
& \left.\mathrm{H}(x) \equiv \frac{\partial h}{\partial x^{\mathrm{T}}}\right|_{x}
\end{aligned}
$$

Then, Prediction of $\hat{x}(k \mid k-1)$ is performed with the time updating procedure $(k=0,1, \cdots)$ as follows:

$$
\begin{aligned}
& \left\{\begin{array}{l}
\hat{x}(k+1 \mid k)=\mathrm{F}(k) \hat{x}(k \mid k)+\bar{u}(k) \\
\hat{x}(0 \mid-1)=\bar{x}_{0}
\end{array}\right. \\
& \left\{\begin{array}{l}
\mathrm{P}(k+1 \mid k)=\mathrm{F}(k) \mathrm{P}(k \mid k) \mathrm{F}(k)^{\mathrm{T}}+\mathrm{U}(k) \\
\mathrm{P}(0 \mid-1)=\mathrm{P}_{0}
\end{array}\right.
\end{aligned}
$$

where the estimation co-variance at $k$ is as follows:

$$
\mathrm{P}(k \mid k)=\Pi_{k}(\hat{x}(k \mid k))
$$

\section{Outline of Gold Sequence}

The Gold sequence is known as sharp autocorrelations and low cross-correlations. As discussed later, it is suitable for simultaneous time-of-flight identifications on many acoustic paths.

The $r$-th Gold sequence, $\left\{v_{K}^{(r)}\right\}$, which consists of binary values, 0 or $1, \quad(r=0, \cdots, N+1 ; \quad K=0, \cdots, N-1)$, is generated with the following way:

$$
\begin{aligned}
& v_{K}^{(0)}=u_{K}, \quad v_{K}^{(1)}=w_{K}, \quad \text { and } \\
& v_{K}^{(r)}=u_{K} \oplus w_{\bmod (K+r-2, N)}, \quad(r=2, \cdots, N+1)
\end{aligned}
$$

where $\left\{u_{K}\right\}$ and $\left\{w_{K}\right\}$ are a preferred pair of the $L$-th order M-sequences with the period, $N=2^{L}-1$. In addition, mod operator performs cyclic shift of the sequence modulo- $N$.

One of the method for obtaining a tally, $\left\{w_{K}\right\}$, of the preferred pair is cyclic sampling from a M-sequence, $\left\{u_{K}\right\}$ as follows:

$$
w_{K}=u_{\bmod (K+q, N)}
$$

where the shifting step, $q=2^{\kappa}+1$, is given by an odd number, $\kappa$, that is mutually prime to the order, $L$. Furthermore, M-sequence, $\left\{u_{K}\right\}$, is recursively generated in the following $L$-th order operation with the exclusive-or, $\oplus$ :

$$
u_{K}=c_{0} u_{K-L} \oplus c_{1} u_{K-L+1} \oplus \cdots \oplus c_{L-1} u_{K-1}
$$

where a proper set of initial values $\left(u_{0}, \cdots, u_{L-1}=0\right.$ or 1$)$ are given, and the coefficients, $c_{K}(K=0, \cdots, L-1)$, are those of a primitive polynomial of the $\mathrm{z}$ operator:

$$
\mathrm{z}^{L}+c_{L-1} \mathrm{z}^{L-1}+\cdots+c_{1} \mathrm{z}+c_{0}=0
$$

Autocorrelations, $\sigma_{r r}(i)$, of the Gold sequences $\left\{v_{K}^{(r)}\right\}$ is evaluated like the impulse, and cross-correlations, $\sigma_{r s}(i)$ $(r \neq s)$, of them take one of three values for all $i$ as follows:

$$
\begin{aligned}
& \sigma_{r r}(i)=\sum_{K=0}^{N-1}\left(1-2 v_{K}^{(r)}\right)\left(1-2 v_{\bmod (K+i, N)}^{(r)}\right) \\
& =m^{(r)} \mathrm{T}^{-i} m^{(r)}=\left\{\begin{array}{l}
N(i=0) \\
-1(i \neq 0)
\end{array}\right. \\
& \sigma_{r s}(i)=\sum_{K=0}^{N-1}\left(1-2 v_{K}^{(r)}\right)\left(1-2 v_{\bmod (K+i, N)}^{(s)}\right) \\
& =m^{(r)} \mathrm{T}^{-i} m^{(s)}=\left\{\begin{array}{l}
-1,1 \pm 2^{\frac{L+1}{2}}(\operatorname{odd} L) \\
-1,1 \pm 2^{\frac{L+2}{2}}(\operatorname{even} L)
\end{array}\right.
\end{aligned}
$$

where the following sequence vector, $m^{(r)}$, and shift operator, $\mathrm{T}$, are defined:

$$
\begin{aligned}
& m^{(r)}=\left(\begin{array}{llll}
m_{N-1}^{(r)} & \cdots & m_{1}^{(r)} & m_{0}^{(r)}
\end{array}\right)^{\mathrm{T}} \text { with } m_{K}^{(r)}=1-2 v_{K}^{(r)} \\
& \mathrm{T}=\left(\begin{array}{cccc}
0 & 1 & \cdots & 0 \\
\vdots & \ddots & \ddots & \vdots \\
0 & \cdots & 0 & 1 \\
1 & 0 & \cdots & 0
\end{array}\right) \in \mathbb{R}^{N \times N}
\end{aligned}
$$

The following $r$-th signals $s_{r}(t)$, of which typical example is shown in Fig.4, with the carrier frequency, $f_{0}$, and the chip width, $d_{c}$, are modulated by the sequences $\left\{v_{K}^{(r)}\right\}:$

$$
s_{r}(t)=a_{r}(t) \cos \left(\omega_{0} t-\frac{\pi}{2}\right)
$$


$\omega_{0}=2 \pi f_{0}=\frac{2 \pi}{d_{c}}$

where $N$ is period of the sequences. $a_{r}(t)$ and $\operatorname{rect}(t)$ are respectively the $r$-th sequence pulse train and the unit rectangular pulse as follows:

$$
\begin{aligned}
& a_{r}(t)=\sum_{K=0}^{N-1}\left\{m_{K}^{(r)} \operatorname{rect}\left(\frac{t}{d_{c}}-K-\frac{1}{2}\right)\right\} \\
& \operatorname{rect}(t)= \begin{cases}1,\left(-\frac{1}{2} \leq t<\frac{1}{2}\right) \\
0, & \text { (otherwise) }\end{cases}
\end{aligned}
$$

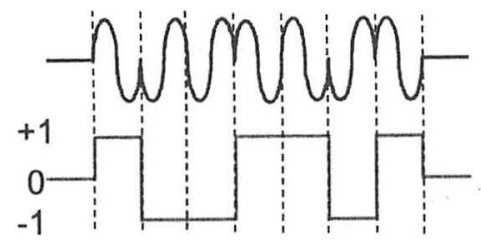

Fig.4 Phase Reversal Keying

\section{Identification of Time-of-flight}

The system function of the acoustic path and transducers, $G(\omega)$, has to be even function, $(G(\omega)=-G(-\omega))$, for the real impulse response, $g(t)$. In addition, their phase shift property, $\theta(\omega)(\equiv-\operatorname{arc}\{G(\omega)\})$, should be regarded as non-linear especially in outdoor environment.

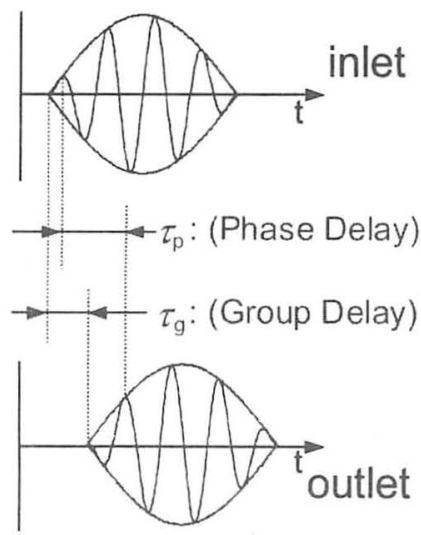

Fig.5 Example of Non-linear Phase Shift

As mentioned in the previous report[1] and shown in Fig.5, the testing wave at the outlet of the path, $s_{\mathrm{R}}(t)$, differs by $\varphi$ in carrier phase from that at the inlet, $s_{\mathrm{T}}(t)$, which consists of carrier frequency, $\omega_{0}$, initial phase, $\phi$, and envelop, $a(t)$, because of the non-linear phase shift:

$$
\begin{aligned}
& s_{\mathrm{T}}(t)=a(t) \cos \left(\omega_{0} t+\phi\right) \\
& s_{\mathrm{R}}(t)=a(t-\tau) \cos \left(\omega_{0}(t-\tau)+\phi+\varphi\right)
\end{aligned}
$$

where the time-of-flight, $\tau$, and additional phase shift parameter, $\varphi$, are given as follows:

$$
\begin{aligned}
& \tau=\tau_{g} \equiv \frac{\mathrm{d} \theta\left(\omega_{0}\right)}{\mathrm{d} \omega}=\frac{\mathrm{d} \theta\left(-\omega_{0}\right)}{\mathrm{d} \omega}, \tau_{p} \equiv \frac{\theta\left(\omega_{0}\right)}{\omega_{0}} \\
& \varphi \equiv \omega_{0} \tau-\theta\left(\omega_{0}\right)=\omega_{0}\left[\tau_{g}-\tau_{p}\right]
\end{aligned}
$$

To apply digital signal processing, the analogue signals are sampled with interval, $\Delta t$. Namely, the signal vector of the $r$ - th acoustic path, $\eta_{r, k} \in \mathbb{R}^{v+1}$, which is degenerated by additive white noise, $\boldsymbol{n}_{k} \in \mathbf{R}^{v+1}$, and carrier phase shift, $\chi=\phi+\varphi$, is supposed to be in the following form:

$$
\eta_{r, k}=\left(\begin{array}{llll}
\eta_{k} & \eta_{k-1} & \cdots & \eta_{k-v}
\end{array}\right)^{\mathrm{T}}=\boldsymbol{V} \boldsymbol{a}_{r}+\boldsymbol{n}_{k}
$$

where the following quantities are defined knowing signal envelop, $a_{r}(t)$, sent to the $r$-th path:

$$
\begin{aligned}
& V=\operatorname{diag}\left\{\cos \left(\Omega_{v}+\chi\right) \quad \cdots \quad \cos \left(\Omega_{1}+\chi\right) \quad \cos \chi\right\} \\
& \Omega_{k}=\frac{2 k \pi}{v+1}(k=1, \cdots, v) \\
& a_{r}=\left(\begin{array}{llll}
a_{r}(v \Delta t) & \cdots & a_{r}(\Delta t) & a_{r}(0)
\end{array}\right)^{\mathrm{T}} \\
& n_{k}=\left(\begin{array}{llll}
n_{k} & n_{k-1} & \cdots & n_{k-v}
\end{array}\right)^{\mathrm{T}}
\end{aligned}
$$

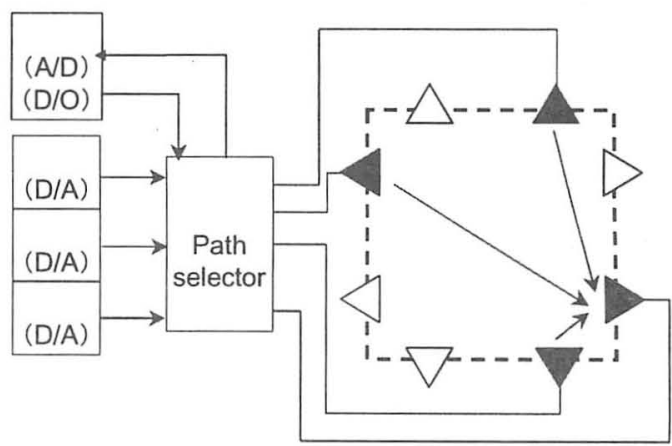

Fig.6 Simultaneous Signal Sending

Suppose without loss of generality that the $v \Delta t$-length signal, $s_{\mathrm{T} r}(t)$, was sent into the $r$-th acoustic path at 0 and was reached the transducer at $\tau=\mu \Delta t$. The properties of the contaminating noise and the signal envelop are assumed as follows: 
$\mathbf{E}\left\{\boldsymbol{n}_{k}\right\}=\mathbf{0}, \mathbf{E}\left\{\boldsymbol{n}_{k} \boldsymbol{n}_{k}^{\mathrm{T}}\right\}=R \boldsymbol{I}_{\boldsymbol{v}+1}$.

For simple discussion, it is further assumed that the chip width, $d_{c}$, of the rectangular pulse in (28) is just $M$ times as the sampling interval, $\Delta t$, and the period of the sequence, $N=2^{L}-1$ is given by odd number, $L$. Thus, it provides as follows:

$$
\begin{aligned}
& a_{r}(k \Delta t)=m_{K}^{(r)}\left(K \leq \frac{k}{M}<K+1, k=0,1, \cdots, v\right) \\
& M=\frac{d_{\mathrm{c}}}{\Delta t}=\frac{v}{N}
\end{aligned}
$$

In order to reduce the total measuring time of the CT system, simultaneous signal sending and time-of-flight identifications on many acoustic paths shown in Fig.6 is applied. Knowing the results of previous report[3], the $\rho$-th optimal processor that detects signal, $s_{\mathrm{R} \rho}(t)$, from the $\rho$-th acoustic path, can be designed with free from carrier phase shift, $\chi$. The response, $\xi_{\rho, r, k}$, of the processor to the signal, $s_{\mathrm{R} r}(t)$, from $r$-th acoustic path, is described in the following form:

$$
\xi_{\rho, r, k}=\left|\left(W a_{\rho}\right)^{*} \eta_{r, k}\right|^{2}
$$

where the complex sinusoidal matrix is defined:

$$
W=\operatorname{diag}\left\{1 \quad \exp \left(\mathrm{j} \Omega_{M}\right) \quad \cdots \quad \exp \left(\mathrm{j} \Omega_{M}\right)\right\}
$$

Then the following expectation of processor response at $v+\mu$ is derived:

$\mathrm{E}\left\{\xi_{\rho, r, v+\mu}\right\}=\mathrm{E}\left\{\left(\boldsymbol{W} a_{\rho}\right)^{*}\left(V a_{r}+n_{v+\mu}\right)\left(V a_{r}+n_{v+\mu}\right)^{*} W a_{\rho}\right\}$

$=\left|\left(\boldsymbol{W} \boldsymbol{a}_{\rho}\right)^{*} V \boldsymbol{a}_{r}\right|^{2}+\boldsymbol{a}_{\rho}^{\mathrm{T}} \boldsymbol{W}^{-1} \mathbf{E}\left\{\boldsymbol{n}_{v+\mu} \boldsymbol{n}_{v+\mu}^{\mathrm{T}}\right\} \boldsymbol{W} \boldsymbol{a}_{\rho}$

$=\frac{1}{4}\left(a_{\rho}{ }^{\mathrm{T}} a_{r}\right)^{2}+R\left|a_{\rho}\right|^{2}=\frac{M^{4}}{4}\left(m^{(\rho) \mathrm{T}} m^{(r)}\right)^{2}+M^{2} R\left|m^{(\rho)}\right|^{2}$

$=\frac{M^{4}}{4}\left(\boldsymbol{m}^{(\rho) \mathrm{T}} \boldsymbol{m}^{(r)}\right)^{2}+R M^{2} N$

Thus, knowing (22) and (23), the value of (44) is evaluated as follows:

$\left|\mathrm{E}\left\{\xi_{\rho, \rho, v+\mu}\right\}\right|=\frac{M^{4} N^{2}}{4}+R M^{2} N$

$\left|\mathrm{E}\left\{\xi_{\rho, r, v+\mu}\right\}\right| \leq \frac{M^{4}\left(1+2^{\frac{L+1}{2}}\right)^{2}}{4}+R M^{2} N \quad(r \neq \rho)$

Namely the ratio of the undesirable response at worst case can be evaluated as follows:

$$
\left|\frac{\mathrm{E}\left\{\xi_{\rho, r, k}\right\}}{\mathrm{E}\left\{\xi_{r, r, p+q}\right\}}\right| \leq \frac{M^{2}\left(1+2^{\frac{L+1}{2}}\right)^{2}+4 R N}{M^{2} N^{2}+4 R N} \approx \frac{1+\frac{2 R}{M^{2}}}{2^{L-1}+\frac{2 R}{M^{2}}}
$$

$$
\left(|k-(v+\mu)|>\frac{M}{2}\right)
$$

where $k$ is not belonging to $\frac{M}{2}$ - neighbor of the maximum and the approximation can be defend at large $L$. The value is typically around 0.1 in $L=7$ case.

\section{Working Examples}

Figure 7 illustrate performance of the proposed signal processor. The received signal which is mixture of three signals from different acoustic paths have identified the time-of-flight \#1 to \#3 with the processor designed for signal \#1 to \#3 respectively applying (42). The result clearly supports the proposed processor that can be applied to $N+1\left(=2^{L}\right)$ signals.

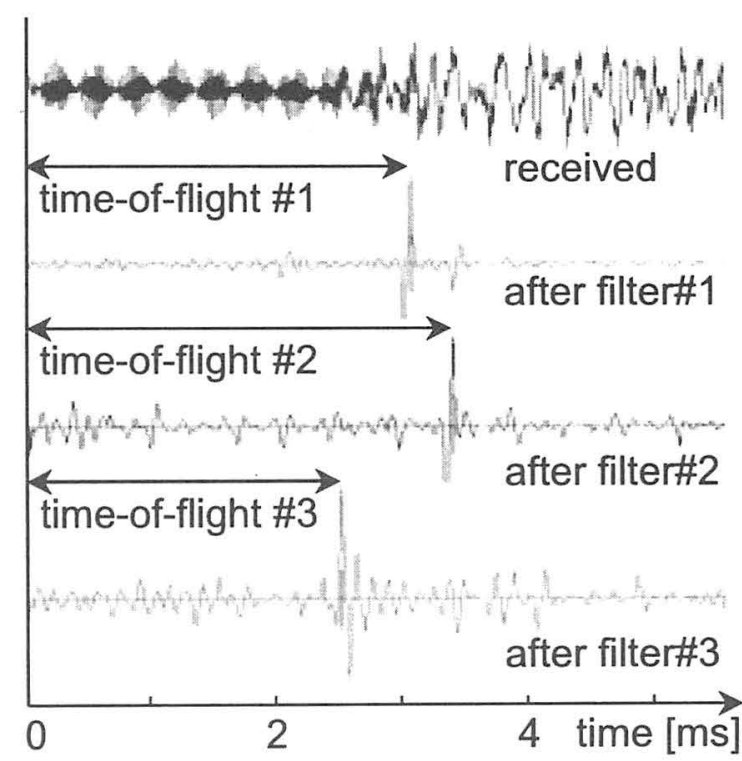

Fig.7 Before and After Signal Processor

Then total performance of proposed CT system for 3-D distribution of gas temperature shall be introduced. Figure 8 shows an example of estimated gas temperature distribution in a duct after inserted a heater. The estimated results before and after heating have been verified with semi-conductor type thermo probes which are set on the points $\mathrm{P}, \mathrm{Q}, \mathrm{R}$ of which coordinate shown in Table 1 . The 
verifications are also summarized in Table 1 and it should be considered to be sufficient.

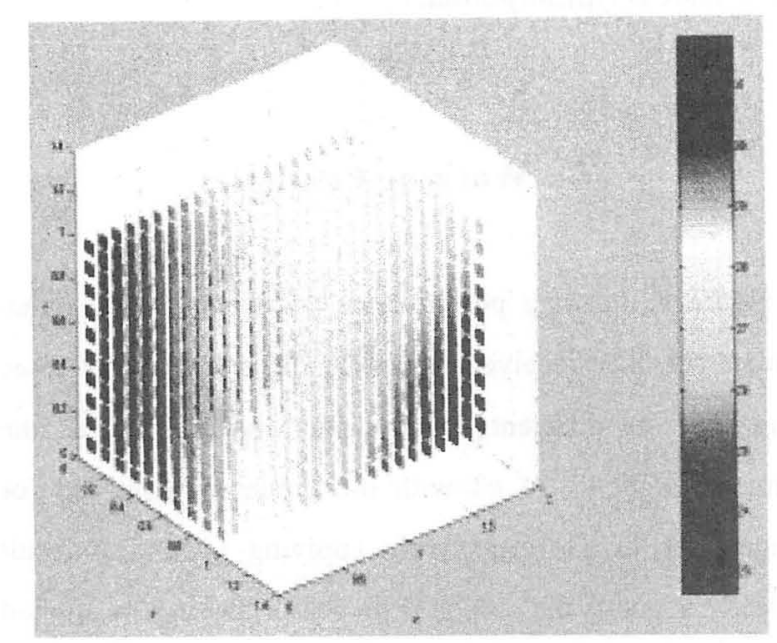

Fig.8 Estimated Gas Temperature Distribution

Table 1 Summary of Verification for Estimated

\begin{tabular}{|c|c|c|c|c|}
\hline \multirow{2}{*}{$\begin{array}{l}\text { Coordinate } \\
(\mathrm{x}, \mathrm{y}, \mathrm{z})[\mathrm{m}]\end{array}$} & \multicolumn{2}{|c|}{ Before } & \multicolumn{2}{|c|}{ After } \\
\hline & $\begin{array}{c}\text { Estimd } \\
{\left[{ }^{\circ} \mathrm{C}\right]}\end{array}$ & $\begin{array}{l}\text { Refer } \\
{\left[{ }^{\circ} \mathrm{C}\right]}\end{array}$ & $\begin{array}{c}\text { Estimd } \\
{\left[{ }^{\circ} \mathrm{C}\right]}\end{array}$ & $\begin{array}{l}\text { Refer } \\
{\left[{ }^{\circ} \mathrm{C}\right]}\end{array}$ \\
\hline $\mathrm{P}(0.2,0.5,0.5)$ & 20.05 & 20 & 29.50 & 30 \\
\hline $\mathrm{Q}(0.2,0.25,1)$ & 20.23 & 20 & 30.41 & 30 \\
\hline $\mathrm{R}(0.2,0.75,1.5)$ & 20.04 & 20 & 26.45 & 26.5 \\
\hline
\end{tabular}

\section{Conclusions}

An acoustic CT system for temperature distributions having the following features has been developed:

1) State estimation with non-linear observation applying MAP estimation with local iteration

2) Signal detection with unknown phase distortion reffering complex absolute

3) Simultaneous sending for time reduction applying the Gold sequence P.R.K.
4) Effectiveness of the proposed method validated

\section{References}

[1] Fukayama, Y. et al.: On signal Processing and Testing Wave for Acoustic Gas Temperature and Flow Measurement; Transaction of SICE, Vol. E-2, pp.139-149 (2002, on http//srv01.sice.or.jp/ e-trans/)

[2] Fukayama, Y. et al.: Acoustic measurement for 3-D distribution of gas temperature and flow applying the extended Kalman filter with local iteration; Proc. of 33rd S.S.S., ISCIE, pp.149-154

[3] Fukayama, Y.: A Study on Acoustic Time-of-flight Identification in Outdoor Environment; Proc. of 35th S.S.S., ISCIE, pp. $85-90$

[4] Proakis, J. G.: Digital Communications; McGraw Hill, New York (1995) 